\title{
Morphological Features of Composite Resin Surfaces after Two- and Three-Body Wear Simulation
}

\author{
Natthavoot Koottathape, Hidekazu Takahashi, Naohiko Iwasaki, Masafumi Kanehira, Werner J Finger
}

\begin{abstract}
Purpose: To investigate morphological surface features of different composite resins after 30,000 sliding wear cycles under two- and three-body wear action.

Materials and methods: The morphology of worn surfaces of four nanofiller containing composite resins, Filtek Supreme XT (FIL), MI Flow (MFL), Venus Diamond (VED) and Venus Pearl (VEP) were investigated and compared with three conventional composites as references, microfilled Durafill VS (DUR), microhybrid Filtek Z250 (Z250) and hybrid-type Clearfil AP-X (APX). Plane surfaces of the polymerized and water-saturated materials $(\mathrm{n}=5$ ) were tested in a custom-built pin-on-disk wear machine fitted with a zirconia ball, $4 \mathrm{~mm}$ in diameter, serving as the antagonist 'cusp' and loading the specimen at $15^{\circ}$ angulation for $3.7 \mathrm{~mm}$ long sliding paths ( $50 \mathrm{~N}$ load, $1.2 \mathrm{~Hz}, 30,000$ cycles) either in water, simulating two-body wear, or in slurries of PMMA beads or poppy seed, simulating three-body wear. Random samples of each material were selected and sputter-coated with gold for inspection of the wear patterns using scanning electron microscopy.
\end{abstract}

Results: Under two-body wear action all materials showed cracking as indicator of fatigue. MFL and VEP with prepolymer particles were least affected. S imilarly, surfaces after three-body wearing with PMMA bead slurry revealed crack formation for five of the composites tested. The micro-hybrid Z250 and the nanofilled FIL displayed compressed and shallow delaminated areas. With poppy seed slurry as the third-body medium DUR was deeply destructed. Also the nanohybrid VED with a small fraction of up to $20 \mu \mathrm{m}$ large ground glass fillers revealed deep cracks, whereas small delamination areas characterized the hybrid composite APX. Z250 as well as FIL, MFL and VEP were smoothly abraded without showing signs of cracking or delamination.

Conclusion: Composite resin surfaces, worn under two-body sliding showed mainly crack formation as an expression of fatigue. Similarly, three-body abrasion with a P MMA bead slurry revealed surface cracks to various extent and delamination, which is also an expression of fatigue related damage. With fine-ground poppy seed slurry only the microfilled composite with large prepolymer particles showed catastrophic failure. In all other cases the small poppy seed fragments in the slurry abraded the surfaces, removing polymer or fine particle loaded matrix uniformly. Supposedly, poppy seed slurry is a suitable third-body medium, simulating occlusal wear produced by food, rich in fibers or grain.

Keywords: Composite resin, Wear, Attrition, Abrasion, Wear pattern.

How to cite this article: Koottathape $\mathrm{N}$, Takahashi $\mathrm{H}$, Iwasaki $\mathrm{N}$, Kanehira M, Finger WJ . Morphological F eatures of Composite Resin Surfaces after Two- and Three-Body Wear Simulation. World J Dent 2012;3(3):221-228.

Source of support: $\mathrm{Nil}$

Conflict of interest: None declared

\section{INTRODUCTION}

In recent dental literature wear of resin composite materials is no longer considered a serious concern, on condition that the occlusal restorations are of small or moderate size and that occlusion and articulation are supported by cavity surrounding enamel. ${ }^{1,2}$ However, in very extended cavities, such as amal gam replacement or when treating patients with bruxing habits, alternative techniques and materials, such as indirectly bonded restorations should be preferred. ${ }^{3-6}$ Long-term clinical trial results are very important to understand wear mechanisms, however the drawback is that materials used in such studies are often no more available in the market.

With the appearance of a considerable number of new nanofiller containing composites and the manufacturers' claims of low polymerization shrinkage, low shrinkagestress and even improved wear resistance, clinical performance data are mostly missing to support these claims. There are only afew published clinical studies comparing micro-hybrid and nano-hybrid composite resins up to 5 or 6 years of service proving that the nano-hybrid types are not superior but rather equivalent to micro- or fine-hybrid composites, ${ }^{4,7-11}$

Therefore, simulating in vitro studies are still desirable or necessary as initial tools to estimate or to predict the clinical performance of such new materials, although full simulation of the many contributing factors in vivo, including patient and operator factors is hardly possible. The oral environment with the many biological variations cannot be properly replicated. Only trends and indications as to the true extent of wear can be obtained. ${ }^{11,12}$

A ny in vitrosimulation should cover the two main wear types occurring on occlusal restorations, namely attrition in the occlusal contact area (OCA, two-body wear) and abrasion in contact-free occlusal area (CFA, three-body wear). ${ }^{13}$ Force, sliding pathways and frequency are among the factors that have to be considered.

Two-body wear is simulated with a cusp at the right dimensions and curvature sliding over the opposing surface in water, or saliva to reduce friction. A s enamel cusp substitutes among others zirconia or steatite balls have been used. ${ }^{14}$ Three-body wear is more difficult to mimic, because abrasion depends on the 'food' bolus that is compressed between cusp and restoration and slides over the surface, where additional abrasion effects might take place, al though 
under decreasing load and friction with increasing distance from the cusp. ${ }^{12}$ Different third-body media have been proposed during the years. Depending upon the third medium different quantitative amount of wear is seen and in particular the morphology of the worn composite largely depends on the abrading food. So far there are only very few, mainly empirical correlations established between in vitro and in vivo wear data, without evidence that the wear patterns are identical or at least similar. ${ }^{14}$

Therefore, it is important to use third-body media that have similar abrasivity as the grinding particles in daily food. Presumably, the main abrasives in food are related to vegetables and grains, where fibers and crushed grain shells are likely the abrading elements. Ignoring this obvious relationship between food and wear, many reports refer to experiments, where SiC powder, PM M A beads, hydroxyapatite slurries, cal cium carbonate slurry, or other empirical mixtures of individual abrasives are used. ${ }^{12}$ One of the crucial determinants is probably the size of the abrasive particles. B eing sufficiently small they can according to the 'protection hypothesis' abrade the interparticle polymer and lead by time to exfoliation of exposed fillers. ${ }^{15,16}$ Thus, the kind of fillers, grain size and distribution and the volume filler loading are important characteristics of composites. Of course, also the polymer and its degree of polymerization must be considered important variables.

$M$ any studies report quantitative wear data, mostly depth of wear, determined by different quantitative methods after varying numbers of load cycles. There is broad consensus, that volume loss is the more relevant parameter. ${ }^{17} \mathrm{~W}$ ithout detailed knowledge about the morphology of the worn surface such data are however, of limited value or even useless in order to understand and explain the underlying wear phenomena.

Therefore, the aim of this invitro trial was to investigate morphological features of different composite resins after 30,000 sliding wear cycles under two- and three-body wear action. The hypothesis to be tested is that there is no difference in morphology of composites worn in water or two alternative third-body slurries.

\section{MATERIALS AND METHODS}

\section{Materials}

Table 1 shows the light-curing composite resin materials investigated. Three materials served as references: DUR is

\begin{tabular}{|c|c|c|c|c|c|}
\hline \multirow[t]{2}{*}{ Materials } & \multirow[t]{2}{*}{ Manufacturer } & \multirow[t]{2}{*}{ Batch } & \multicolumn{3}{|c|}{ Composition } \\
\hline & & & Monomer & Filler & Vol(\%) \\
\hline $\begin{array}{l}\text { Durafill }{ }^{\circledR} \\
\text { VS (DUR) }\end{array}$ & $\begin{array}{l}\text { Heraeus Kulzer, } \\
\text { Germany }\end{array}$ & 010210 & $\begin{array}{l}\text { Bis-GMA, } \\
\text { UDMA, } \\
\text { TEGDMA }\end{array}$ & $\begin{array}{l}\mathrm{SiO}_{2}(20-70 \mathrm{~nm}) \text { and } \\
\text { prepolymer }<20 \mu \mathrm{m}\end{array}$ & 66 \\
\hline $\begin{array}{l}\text { Clearfill } \\
\text { AP -X } \\
(A P X)\end{array}$ & Kuraray, J apan & $1078 A$ & $\begin{array}{l}\text { Bis-GMA } \\
\text { TEGDMA }\end{array}$ & $\begin{array}{l}\text { Silinated barium glass, } \\
\text { silinated colloidal silica, } \\
\text { silinated silica }(0.1-15 \mu \mathrm{m})\end{array}$ & 70 \\
\hline $\begin{array}{l}\text { Filtek }^{\mathrm{TM}} \\
\text { Z250 } \\
(\mathrm{Z} 250)\end{array}$ & $3 M$ ESPE, USA & 9UR & $\begin{array}{l}\text { Bis-GMA } \\
\text { Bis-EMA } \\
\text { UDMA }\end{array}$ & $\begin{array}{l}\mathrm{SiO}_{2}, \mathrm{ZrO}_{2} \text { clusters, } \\
\text { average } 0,6 \mu \mathrm{m} \\
(0.19-3.3 \mu \mathrm{m})\end{array}$ & 60 \\
\hline $\begin{array}{l}\text { Filtek }^{T M} \\
\text { Supreme } \\
\text { XT (FIL) }\end{array}$ & $3 M$ ESPE, USA & $9 \mathrm{WT}$ & $\begin{array}{l}\text { Bis-GMA, } \\
\text { UDMA, } \\
\text { Bis-EMA, } \\
\text { TEGDMA }\end{array}$ & $\begin{array}{l}\mathrm{SiO}_{2} / \mathrm{SrO}_{2} \text { clusters }(0.8-1,4 \mu \mathrm{m}) \text {, } \\
\mathrm{SiO}_{2}(20 \mathrm{~nm})\end{array}$ & 59.5 \\
\hline $\begin{array}{l}\text { MI Flow } \\
(M F L)\end{array}$ & GC, J apan & 0904132 & $\begin{array}{l}\text { UDMA, } \\
\text { Bis-MEPP, } \\
\text { DMA }\end{array}$ & $\begin{array}{l}\text { Silica- (16 } \mathrm{nm}) \text { and Sr-doped } \\
\text { nanofiller }(700 \mathrm{~nm}) \text {, lanthanoid } \\
\text { fluoride }(100 \mathrm{~nm})\end{array}$ & 50 \\
\hline $\begin{array}{l}\text { Venus }^{\circledR} \\
\text { Diamond } \\
\text { (VED) }\end{array}$ & $\begin{array}{l}\text { Heraeus Kulzer, } \\
\text { Germany }\end{array}$ & 010027 & $\begin{array}{l}\text { TCD-DI-HEA, } \\
\text { UDMA }\end{array}$ & $\begin{array}{l}\text { Ba-Al-F-Si glass }<20 \mu \mathrm{m}, \\
\mathrm{SiO}_{2} \text { nanofiller }(5 \mathrm{~nm})\end{array}$ & 64 \\
\hline \multicolumn{6}{|l|}{ Venus } \\
\hline $\begin{array}{l}\text { Pearl } \\
\text { (VEP) }\end{array}$ & $\begin{array}{l}\text { Heraeus Kulzer, } \\
\text { Germany }\end{array}$ & VP 301110 & $\begin{array}{l}\text { TCD-DI-HEA, } \\
\text { UDMA }\end{array}$ & $\begin{array}{l}\text { Ba-Al-F-glass, prepolymerized } \\
\text { filler, } \mathrm{SiO}_{2} \text { nanofiller } \\
\text { (grain size: } 5 \mathrm{~nm} \text { to } 5 \mathrm{~nm} \text { ) }\end{array}$ & $\begin{array}{l}59 * \\
(* 58 \% \text { in } \\
\text { organic) }\end{array}$ \\
\hline
\end{tabular}

Abbreviation: Bis-GMA: Bisphenol A diglycidylether methacrylate; $B$ is-MEPP: 2,2-Bis (4-methacryloxypolyethoxyphenyl) propane; UDMA: Urethane dimethacrylate; TEGDMA: Triethylene glycol dimethacrylate; Bis-EMA: E thoxylated bisphenol-A dimethacrylate; DMA: Dimethacrylate; TCD-DI-HEA: Bis-(acryloyloxymethyl) tricyclo $(5.2 .1 .02,6)$ decane 

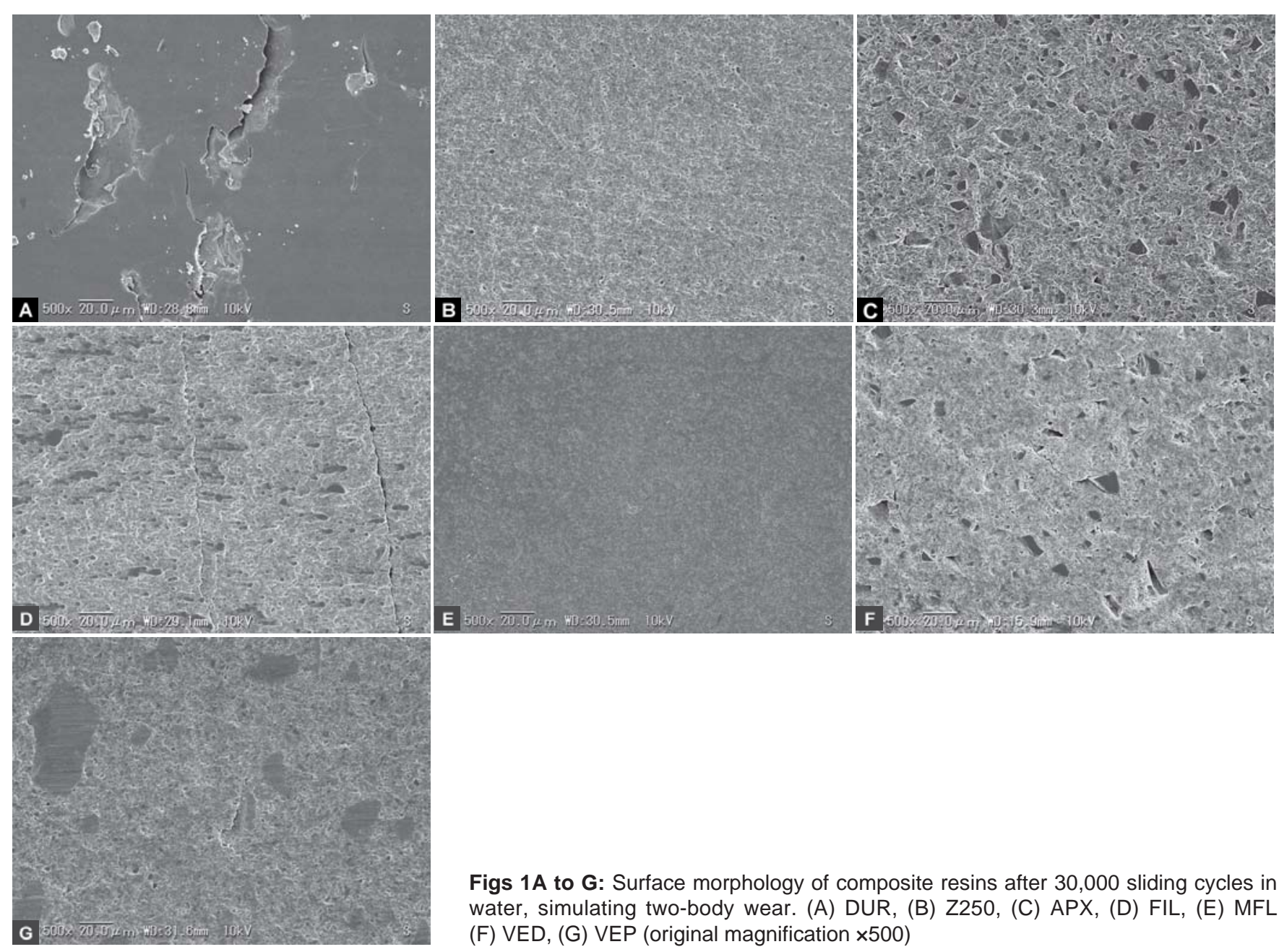

Figs $\mathbf{1 A}$ to $\mathbf{G}$ : Surface morphology of composite resins after 30,000 sliding cycles in water, simulating two-body wear. (A) DUR, (B) Z250, (C) APX, (D) FIL, (E) MFL $(F)$ VED, (G) VEP (original magnification $\times 500$ )

a microfilled (A 3), APX a hybrid (A 2) and Z250 a microhybrid (A 3) type. FIL is a nanofilled (A 3B) composite, M FL a flowable nano-hybrid (A 3), VED and VEP arenano-hybrid (A 3 ) composite resins with the same monomer composition yet different filler concepts.

\section{Specimen Preparation}

All composite specimens were produced at ambient laboratory atmosphere in aluminum molds, $8 \mathrm{~mm}$ in diameter and $2 \mathrm{~mm}$ in depth. Overfilled cavities were covered with a Mylar strip and pressed flush. Light activation for 40 seconds with a QTH curing unit ( $X$ L 3000, 3M ESPE, M N , USA; output $>500 \mathrm{~mW} \mathrm{~cm}^{-2}$ ). I mmediately after curing, the specimens were immersed in $37^{\circ} \mathrm{C}$ water and stored for 7 days. Then, excess was removed by manually grinding on wet SiC paper, grits \#600, 1500 and 4000.

\section{Wear Testing Device}

A custom-made ball-sliding on disk wear testing machine fitted with a zirconia ball, $4 \mathrm{~mm}$ in diameter serving as the antagonist 'cusp' was used, loading the composite specimen at $15^{\circ}$ angulation. At the end of each $3.7 \mathrm{~mm}$ long sliding path motion the zirconia ball was lifted and returned into the zero position for the next sliding cycle ( $50 \mathrm{~N}$ load, 1.2 $\mathrm{Hz}, 30,000$ cycles). The composite specimens were mounted in a plexiglass container, filled with water for simulation of two-body wear, an aqueous slurry of 30 mass $\%$ polymethyl methacrylate (PM M A : denture base material polymer beads, Palapress, Heraeus K ulzer, Germany, average particle size: $40 \mu \mathrm{m}$ ) or an aqueous slurry of 33 mass $\%$ lightly pre-ground poppy seeds for simulation of three-body wear, respectively. For each resin composite 5 specimens were tested under each of the three conditions, using freshly prepared abrasive slurries after each 10,000 sliding cycles. All testing was done at ambient atmosphere $\left(23 \pm 2^{\circ} \mathrm{C}, 50 \pm 10 \% \mathrm{RH}\right)$.

\section{Scanning Electron Microscopy (SE M)}

Representative specimens of each material after each of the wear test conditions w ere selected, sputter-coated with gold to a thickness of approximately $5 \mathrm{~nm}$ and examined by SEM (VE-8800, Keyence Corp., Osaka, Japan) under $10 \mathrm{kV}$ acceleration. Furthermore, scanning electron microphotographs were made from the third-body media PM M A and poppy seed before and after use for 10,000 wear cycles. 


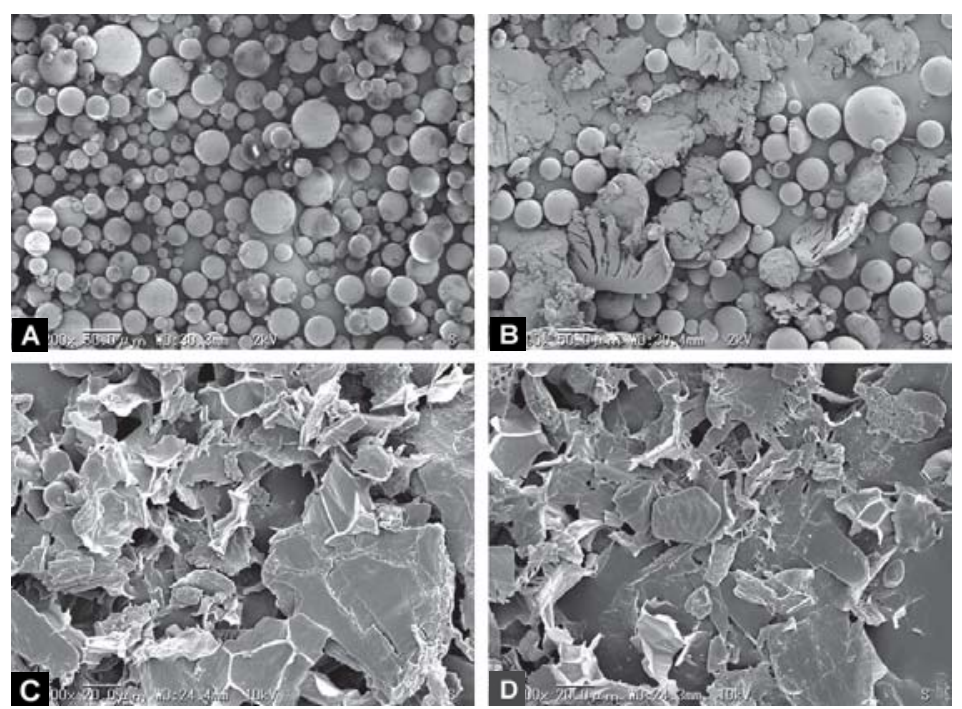

Figs 2A to D: PMMA beads before $(A)$ and after $(B)$ use (original magnification $\times 200$ ) and milled poppy seed before (C) and after (D) use (original magnification $\times 500$ ) as third-body abrasion slurry for 10,000 wear cycles
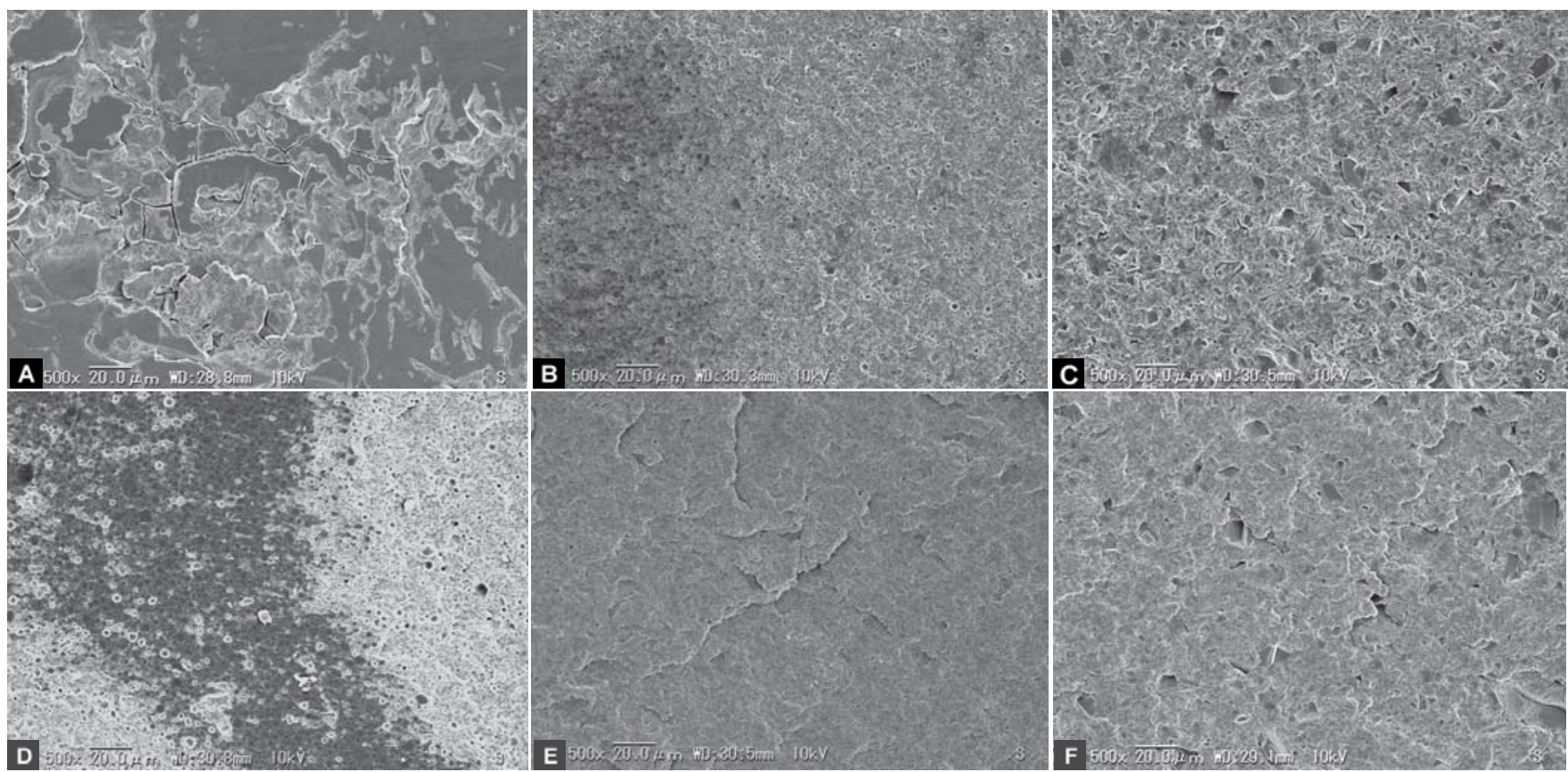

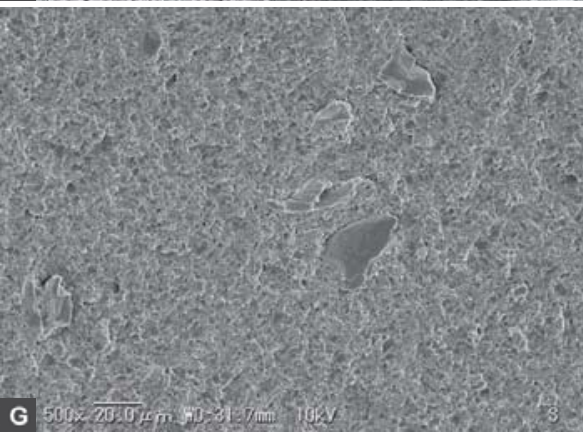

\section{RESULTS}

The SEM microphotographs (Figs $1 A$ to $G$ ) show representative areas for each composite from the final thirds of the sliding tracks at 500-fold magnification, following wearing in the attrition mode, i.e. in water. On all photographs the ball sliding direction was from right to left.
Figs 3A to G: Surface morphology of composite resins after 30,000 sliding cycles in PMMA bead slurry, simulating three-body wear. (A) DUR, (B) Z250, (C) APX, (D) FIL, (E) MFL, (F) VED, (G) VEP (original magnification $\times 500$ )

The microfilled DUR (Fig. 1A) was mostly characterized by a smooth surface. However, numerous cracks associated with local defects were seen. These cracks extended perpendicular to the sliding direction along the interface between the prepolymerized particles and matrix polymer. Z250 (Fig. 1B) was rather uniformly worn; multiple small 
Morphological Features of Composite Resin Surfaces after Two- and Three-Body Wear Simulation

holes on the surface were seen resulting from exfoliation of spherical filler particles. Moreover, perpendicular to the sliding direction a rhythmic pattern of small cracks was displayed. The hybrid type composite APX (Fig. 1C) showed numerous up to $20 \mu \mathrm{m}$ large glass fillers. M any of these fillers were slightly extending over the surrounding specimen-surface, some were loosened or had lost their grip in the polymer. Initial cracking through the polymer loaded with finer glass particles was seen. The nanofilled FIL (Fig. 1D) was smoothly abraded, long gaps were prominent at right angle to the sliding direction. A part from tiny holes left after debonding of nanofiller clusters numerous clusters were abraded to the level of the neighboring polymer. The horizontal dark areas were apparently polymer-rich islands. M FL (Fig. 1E) was very uniformly abraded, only few small pit holes were detected. The worn VED (Fig. 1F) surface show ed characteristically scattered large glass particles and holes after pluck-out of fillers. Typically, as illustrated at the large central particle, cracks originated from sharp edges and extended in various directions. In contrast, the large prepolymer particles included in VEP (Fig. IG) were mostly well-retained in surrounding polymer. Scratches were displayed in sliding direction. The more fine-grained fillers in the bulk of the composite were apparently well-anchored in matrix polymer.

Figures $2 A$ to $D$ showed PM M A beads before (Fig. 2A) and after using in slurry (Fig. 2B) as third-body medium. The nonloaded beads were perfectly spherical, whereas a number of polymer beads were crushed after 10,000 sliding cycles. The pre-ground and the poppy seeds used in the slurry (Figs $2 \mathrm{C}$ and D) were very similar. They showed laminated slab-like appearance next to thin fibrous elements.

The SEM S in Figures $3 \mathrm{~A}$ to $\mathrm{G}$ demonstrate the morphology of composite surfaces after 30,000 sliding cycles with PMMA slurry as third-body medium. Characteristic for DUR (Fig. 3A) were very smoothly worn areas next to locations with extensive cracking, shallow surface defects and broken loose parts of prepolymer particles. Z250 (Fig. 3B) was very uniformly abraded. M any pit holes indicated loss of small filler particles. Sporadic tiny cracks were visible. Very similar to the appearance of APX after two-body wearing the surface after three-body loading with PM M A beads (Fig. $3 \mathrm{C}$ ) show ed exposed large glass fillers, both retained and loosened. B eds of exfoliated fillers as well as broken glass particles were seen. The FIL (Fig. 3D) surface demonstrated scattered areas rich in
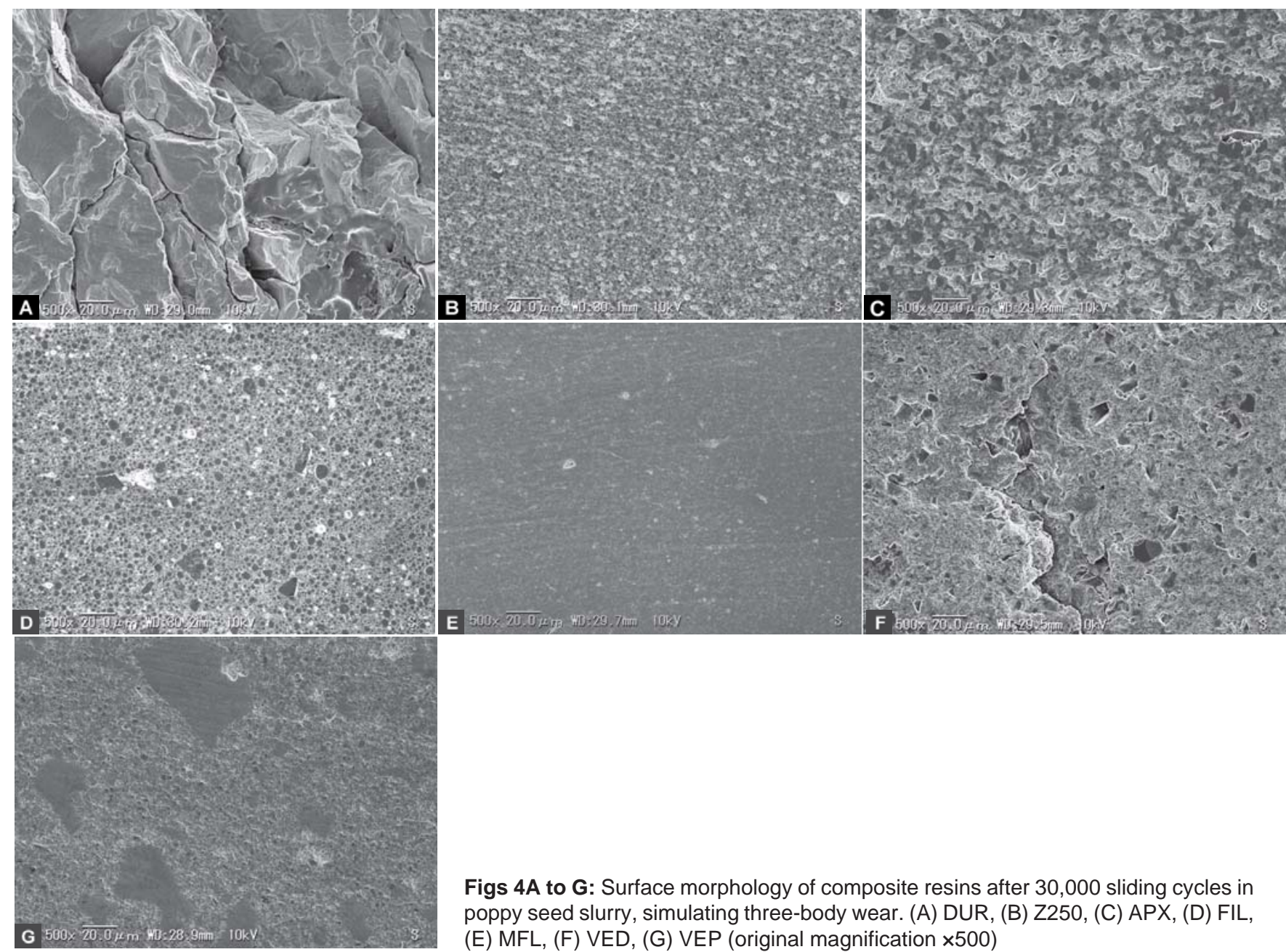

Figs 4A to G: Surface morphology of composite resins after 30,000 sliding cycles in poppy seed slurry, simulating three-body wear. (A) DUR, (B) Z250, (C) APX, (D) FIL, (E) MFL, (F) VED, (G) VEP (original magnification $\times 500$ ) 
polymer (dark) with persisting abraded clusters and areas characterized by predominantly shallow surface destruction (white) where a lot of pits after cluster debonding were prominent. MFL (Fig. 3E) had a stacked or horizontally stratified surface, typically laminated and cracked along the straticulate layers. The VED surface (Fig. 3F) illustrated, as the surface after attrition loading, broken and lost large fillers and multiple tiny cracks perpendicular to the sliding direction of the zirconia ball. $L$ arge prepolymer particles in VEP (Fig. 3G) were rocked loose or fractured. The bulk of the polymer matrix displayed similar cracking tendency as seen with VED.

A fter 30,000 wear cycles in poppy seed slurry (Figs 4A to $G$ ) the micromorphology of the worn composite surfaces was distinctly different from attrition or PM M A-loading wear. DUR (Fig. 4A) exhibited a plurifragmentary fracture. This catastrophic failure site showed wreckage from deep fractures through both prepolymer and matrix polymer. In contrast Z250 (Fig. 4B) was apparently only superficially worn. Grinding scratches, presumably caused by parts of the poppy seed shells were seen in sliding direction. Only detached pits left after filler loss were illustrated. Even the hybrid-type APX (Fig. 4C) was rather uniformly abraded under the action of the poppy slurry. Only strayed filler losses were recognized, whereas some areas demonstrated superficial loss of matrix polymer including fine-grained filler particles. FIL (Fig. 4D) showed a predominantly even surface. Beds after lost clusters were scarcely found. M ost clusters seemed worn at a level with the surrounding matrix polymer. MFL (Fig. 4E) was very smoothly abraded. Scratching grooves in sliding direction were likely produced by poppy shell fragments. In case of VED (Fig. 4F) several deep cracks dominated the appearance. As under the alternative abrasion modes, large fillers were exfoliated, others protruded from the surroundings, either still securely anchored in or already loosened from the polymer matrix. VEP (Fig. 4G) impressed by uniform wear through both the filler-rich matrix and the comparatively large prepolymer particles, displaying tiny grooves from the abrasive medium. The prepolymer fillers were securely bonded to the surrounding matrix polymer.

\section{DISCUSSION}

The hypothesis to be tested, that there is no difference in morphology of composites worn in water or two al ternative third-body slurries has to be rejected.

In general, wear may be due to several different mechanisms, either individual or in combination, including adhesive, abrasive, fatigue and corrosive events. ${ }^{18}$ The dominating wear mechanism on occlusal composite restorations depends on the area, either OCA, occlusal contact area, that is normally referred to as attrition, or CFA, the contact-free area, mostly referred to as abrasion zone. Fatigue and abrasion contribute to attrition, whereas abrasion is the dominating mechanism in CFA. A ttrition is attributed to opposing tooth contact and considered a localized process mainly resulting from local microfractures. W ear in CFA occurs in a three-body wear mode, i.e. under sliding motion of the antagonist cusp transmitting force through a food bolus that serves as the third-body medium. ${ }^{9}$ B oth of the loading conditions were simulated in the present trial.

A nalysis of the wear patterns produced under these conditions is essential for understanding of the wear mechanisms involved. Attrition effects on the materials studied were distinctly different. When the zirconia ball slides over the DUR surface, due to plastic deformation a zone of compression is established in front of the ball and a zone of tension behind. W ith repeated numbers of cycles the energy is dissipated, leading to crack formation perpendicular to the sliding direction and propagating predominantly around the weak interface between the prepolymer particles and the matrix. ${ }^{19,20}$ Finally, chip fractures occur along the pathway of the cracks.

The morphological appearances of Z250, FIL and M FL after two-body sliding wear were similar. Presumably, due to the relatively high filler loading and the small average filler grain size of these composites, after initial wearing the force transmitted of the zirconia ball is distributed over a large area, where many filler particles are the bearing elements, thus dissi pating stress. The greater the number of bearing filler particles on the loaded surface, the larger is the contact area between particles and antagonist, hence the better the wear resistance. ${ }^{21}$ Small crack zones perpendicular to the sliding direction were seen pointing to fatigue mechanisms. In case of A PX and VED a relatively small number of larger particles protruded through the surface and were apparently subjected to a combination of normal loading and frictional shear forces. With increase in cantilever length these fillers are prone to be pulled out of the surface. They can thus, easily befractured or dislodged from the surrounding polymer. With increase in filler loosening fatigue cracks originating from sharp corners (Fig. 1F, VED) were readily initiated. In case of VEP, however, the larger fillers are prepolymer particles with a Y oung's modulus similar to the neighboring matrix. Thus, they absorb the imposed stress much better and the composite is less prone to debonding or cracking.

A ccording to $\mathrm{Hu}$ et $\mathrm{al}^{22}$ the largest effect on occlusal composite wear is related to three-body wear. 
Morphological Features of Composite Resin Surfaces after Two- and Three-Body Wear Simulation

Figures $3 A$ to $G$, illustrate the surface patterns after threebody wear using PM M A-slurry. Seemingly,under the load of the sliding zirconia ball the PMMA beads acted as particles transmitting high local force on elements of the composite surface. Large PM M A beads were crushed and might therefore act as scratching fragments during the continued wear process. A s under two-body sliding the wear pattern of DUR showed typical signs of fatigue with gross local crack formation. When initial fatigue cracks propagate, they eventually coalesce in a plane horizontally under the free surface. ${ }^{22}$ This phenomenon might explain the delaminated areas seen next to the smooth compressed sites. A lso the wear patterns of Z250 (Fig. 3B) and FIL (Fig. 3D) revealed signs of delamination, which may be described as layer-by-layer abrasion. In both materials spherical filler particles were removed to a large extent in the del aminated zone, whereas most of the filler particles remained intact in the compressed areas. The flowable composite M FL with $200 \mathrm{~nm}$ average grain size was seriously damaged under the action of the PMMA beads, acting locally as force transmitting balls with very small diameter. The layer-bylayer cracking effect was very pronounced. The wear pattern morphologies of A PX and VED remind of the wear patterns seen under two-body loading. The hard protruding glass particles served as asperities that were loosened, cracked or exfoliated under the force of the zirconia antagonist ball. The PM M A beads had probably only a minor effect. U sing PM MA beads in aqueous slurry as third body abrasion medium is probably not suitable to simulate in vivo wear in contact-free areas.

Under the action of poppy seed slurry as third-body medium the appearance of the worn surfaces' morphology was much different. During sliding the wear surfaces were separated from each other by the slurry, distributing the load over a larger area. It is supposed that the wear seen was primarily a result of abrasion caused by the crashed poppy seed shells and fibers (Figs $2 C$ and D). Such grinding particles are likely to scratch the matrix polymer phase between fillers, or the matrix enriched with very fine-grained filler particles, an assumption that is supported by the protection theory. ${ }^{15,16}$ For theoretical reasons composites loaded with fine-grained filler particles would therefore show higher wear resistance under food abrasion than under the impact of attrition wearing. DUR was severely damaged, probably as an effect of microcrack propagation around the prepolymer blocks. The microcrack front may continue to grow deeper into the material and finally lead to the appearance show $n$ in Figure 4A, a severe w reckage, which might be an expression for the catastrophic failure phenomenon found in clinical studies after several years in service. ${ }^{23,24}$ For composites with rather large glass fillers, such as APX and VED the poppy seed slurry may grind the resin matrix between the rather fine-grained fillers away, eventually exfoliating particles and leaving a resin-rich, unprotected layer that is again prone to be abraded. Thus, with time the larger fillers will protrude more and more above the surface where they eventually are loaded by the antagonist 'cusp' and rocked loose (Figs 4C and F). Very similar as under two-body wear, cracks would initiate from sharp corners or edges of such large fillers, propagate and result in deeper fatigue cracking ( $\mathrm{Fig}$. 4F). This assumption is supported by the morphological appearance of VEP (Fig. $4 G$ ), a material similar to VED, yet with prepolymerized large particles that were quite uniformly abraded, al most simultaneous with the surrounding glass filler loaded composite. No fatigue-like cracks were observed for VEP. For composites with small filler sizes wear caused by poppy seed slurry became more uniform, as demonstrated with Z250 (Fig. 4B) and FIL (Fig. 4D). Only few pit holes were observed. Unlike the spherical glass fillers in Z250, FIL contains aggregated nanofiller clusters that are eventually interpenetrated with resin. A s the surface of such clusters is subjected to abrasion the small nanofillers that make up the clusters tend to break apart rather than debond entirely. ${ }^{9}$ Therefore, a very uniform wear pattern was observed. A similarly smooth and uniform abrasion pattern was seen with the flowable M FL, a composite containing $200 \mathrm{~nm}$ fillers in apparently very narrow grain size distribution.

\section{CONCLUSION}

The present assessment of composite surfaces, worn under two-and three-body abrasion, revealed morphological details that were related to fatigue mechanisms and abrasive wear. Considering three-body loading, which is supposedly the main mechanism causing wear during clinical service of occlusal restorations, it is crucial to select an appropriate third-body medium as food-like slurry. Evaluation of composite surfaces' micromorphology after several-year service in vivo would be highly desirable, to compare with the morphology of in vitro produced wear patterns of composite resins resulting from different third-body media. Such observations together with quantifiable wear data would offer new gateways to design of laboratory wear methods and understanding of wear mechanisms.

\section{ACKNOWLE DGMENT}

The authors gratefully acknowledge donation of proprietary composite resin materials from GC Corporation, J apan and Heraeus Kulzer, Germany. 


\section{REFERENCES}

1. Ferracane JL. Resin composite: State of the art. Dent M ater 2011;27:29-38

2. Ferracane JL. Is the wear of dental composites still a clinical concern? Is there still a need for in vitro wear simulating devices? Dent $M$ ater 2006;22:689-92.

3. V an Nieuwenhuysen JP, D'HooreW, Carvalho J, Quist V. L ongterm evaluation of extensive restorations in permanent teeth. J Dent 2003:31:395-405.

4. K rämer N, Garcia-Godoy F, R einelt C, Feilzer A J N Nanohybrid vs fine hybrid composite in extended class II cavities after 6 years. Dent $M$ ater 2011;27:455-64.

5. Van Dijken JW. Direct resin composite inlays/onlays: An 11 year follow-up. J Dent 2000;28:299-306.

6. Pallesen $U$, Quist V. Composite resin fillings and inlays: An 11-year evaluation. Clin Oral Invest 2003;7:71-79.

7. Ernst CH, Brandenbusch CM , M eyer G, Canbek K, Gottschalk $\mathrm{F}$, Willershausen $\mathrm{B}$. Two-year clinical performance of a nanofiller vs a fine-particle hybrid resin composite. Clin Oral Invest 2006;10:119-25.

8. K rämer $\mathrm{N}$, R einelt $\mathrm{C}$, R ichter $\mathrm{G}$, Petschelt $\mathrm{A}$, F rankenberger $\mathrm{R}$. Nanohybrid vs fine hybrid composite in Class II cavities: Clinical results and marginal analysis after four years. Dent $M$ ater 2009;25:750-59.

9. Y esil ZD, A lapati S, J ohnston W, Seghi RR. Evaluation of the wear resistance of new nanocomposite resin restorative materials. J Prosthet Dent 2008;99:435-43.

10. Palaniappan S, B haradwaj D, Mattar DL, Peumans M, van $M$ eerbeek $B, L$ ambrechts $P$. Three-year randomized clinical trial to evaluate the clinical performance and wear of a nanocomposite versus a hybrid composite. Dent M ater 2009;25:1302-14.

11. Palaniappan S, B haradwaj D, M attar DL, Peumans $M$, van Meerbeek B, Lambrechts P. N anofilled and microhybrid composite restorations: Five-year clinical wear performances. Dent $M$ ater 2011:27:692-700.

12. Lambrechts $P$, Debels $E$, van Landuyt $K$, Peumans $M$, van M eerbeek B. How to simulate wear? Overview of existing methods. Dent M ater 2006:22:693-701.

13. ISO dental materials-guidance on testing of wear. Part 2 . W ear by two- and/or three-body contact. Technical Specification 2001;14569-72.

14. HeintzeSD. How to qualify and validate wear simulation devices and methods. Dent $M$ ater 2006;22:712-34.

15. Bayne SC, Taylor DF, H eymann HO. Protection hypothesis for composite wear. Dent M ater 1992;8:305-09.

16. Jørgensen K D, Hørsted P, J anum O, K rogh J, Schulz J. A brasion of class I restorative resins. Scand J Dent Res 1979;87:140-45.

17. DeL ong R. Intraoral restorative materials wear: Rethinking the current approaches: How to measure wear. Dent M ater 2006;22:702-11.

18. Mair LH, Stolarski TA, Vowles RW, Lloyd CH. Wear: $M$ echanisms, manifestations and measurement. Report of a workshop. J Dent 1996;24:141-48.

19. Y ap AUJ, Teoh SH, Chew CL. Effects of cyclic loading on occlusal contact area wear of composite restoratives. Dent $M$ ater 2002;18:149-58.
20. W assell RW, M cCabe JF, W alls A W. W ear characteristics in a two-body wear test. Dent M ater 1994;10:269-74.

21. Turssi CP, Ferracane J L, V ogel K. Filler features and their effects on wear and degree of conversion of particulate dental resin composites. Biomaterials 2005;26:4932-37.

22. $H u X, M$ arquis $P M$, Shortall A C. Influence of filler loading on the two-body wear of a dental composite. J Oral Rehabil 2003;30:729-37.

23. L ambrechts $P, B$ raem $M, V$ anherle $G$. Evaluation of clinical performance for posterior composite resins and dentin adhesives. Oper Dent 1987;12:53-78.

24. Tyas M J, W assenaar P. Clinical evaluation of four composite resins in posterior teeth: Five-year results. A ust Dent J 1991;36:369-73.

\section{ABOUT THE AUTHORS}

\section{Natthavoot Koottathape}

Graduate Student, Department of A dvanced B iomaterials, Graduate School of M edical and Dental Sciences, Tokyo M edical and Dental University, Tokyo, Japan

\section{Hidekazu Takahashi}

Professor and Chair, Oral Biomaterials Engineering, Course for O ral Health Engineering, School of O ral Health Care Sciences, Faculty of Dentistry, Tokyo M edical and Dental U niversity, Tokyo, Japan

\section{Naohiko Iwasaki}

A ssistant Professor, Oral Biomaterials Engineering, Course for Oral Health Engineering, School of O ral Health Care Sciences, Faculty of Dentistry, Tokyo M edical and Dental U niversity, Tokyo, Japan

\section{Masafumi Kanehira}

Assistant Professor, Department of Restorative Dentistry, Division of Operative Dentistry, Tohoku U niversity Graduate School of Dentistry, Sendai, J apan

\section{Werner J Finger}

Guest Professor, Department of Restorative Dentistry, Division of O perative Dentistry, Tohoku U niversity G raduate School of D entistry Sendai, J apan

\section{CORRESPONDING AUTHOR}

Hidekazu Takahashi, Professor and Chair, Oral Biomaterials Engineering, Course of Oral Health Engineering, School of Oral Health Care Sciences, Faculty of Dentistry, Tokyo Medical and Dental University, 1-5-45, Y ushima B unkyo-ku, Tokyo 113-8549, Japan, Phone: 81-3-5803-5379, Fax: 81-3-5803-5379 e-mail: takahashi.bmoe@tmd.ac.jp 